\title{
Kinetic Limitations in Electronic Growth of Ag Films on Fe(100)
}

\author{
K. L. Man, ${ }^{1}$ Z. Q. Qiu, ${ }^{1,2}$ and M. S. Altman ${ }^{1}$ \\ ${ }^{1}$ Department of Physics, Hong Kong University of Science and Technology, Clear Water Bay, Kowloon, Hong Kong \\ ${ }^{2}$ Department of Physics, University of California at Berkeley, Berkeley, California 94720, USA
}

(Received 30 June 2004; published 2 December 2004)

\begin{abstract}
Quantum well (QW) resonances are identified in $\mathrm{Ag}$ films on an $\mathrm{Fe}(100)$ surface and are used in low energy electron microscopy to monitor film morphology during annealing and growth. We find that $\mathrm{Ag}$ films thermally decompose to thicknesses that are stabilized by QW states at the $\bar{\Gamma}$ point. Novel growth morphologies are also observed that highlight the competition between kinetic limitations and the QW state energetics that promote electronic growth. These combined observations help to explain the unusual bifurcation mode of thermal decomposition that was reported previously for this system.
\end{abstract}

DOI: 10.1103/PhysRevLett.93.236104

The properties of ultrathin metal films exhibit remarkable quantum size effects (QSE) due to the discrete quantum well $(\mathrm{QW})$ states that are caused by electron confinement [1]. Considerable early interest in metallic QW states was focused on their role in oscillatory magnetic interlayer coupling [2]. It has also been established recently that QW states can have a dramatic influence on film morphology [3-13] in the same nanoscale thickness regime that magnetic coupling and QSE in other properties, such as work function [14], thermal desorption [15], and interlayer relaxations [8], are observed. In particular, it was found that film morphology can be dominated during growth or annealing by film thicknesses that support strongly bound QW states at the $\bar{\Gamma}$ point, which induce total energy local minima compared to other thicknesses. This ideally occurs with the same periodicity in film thickness that QW states cross the Fermi level. However, a beating of the QW period and the discrete layer spacing is sometimes evident in film morphology [3,11]. The consideration of QW state energetics has therefore accounted for a variety of multiple layer height features in the film morphologies of several systems. This phenomenon, which is often referred to as electronic growth, is envisioned to be a mechanism for self-organization in fabrication.

A particularly interesting example of electronic growth was seen in the thermal stability of atomically flat $\mathrm{Ag}$ films on the $\mathrm{Fe}(100)$ surface [12]. With the exception of $N=2$ and $N=5$ monolayer (ML) thick $\mathrm{Ag}$ films, which were stable during annealing up to high temperature, an initially uniform $N$ ML thick film was found to transform, or bifurcate, to a combination of $N-$ 1 and $N+1 \mathrm{ML}$ thick regions upon slight annealing above room temperature. This unusual behavior was attributed to the shape of the local energy versus thickness landscape defined by QW states in $N-1, N$, and $N+1$ film thicknesses. While the stability of $5 \mathrm{ML}$ thick $\mathrm{Ag}$ films was clearly related to the existence of a QW state far below the Fermi level, the energy landscape has negative curvature between QW-induced energy minima $[12,13]$.
PACS numbers: 68.55.Jk, 68.37.Nq, 68.55.Ac, 73.21.Fg

Thus, for these energetically unfavorable film thicknesses, the system may lower its total energy through bifurcation. The surprising aspect of this result was that an initially uniform $N$ ML thick film $(N \neq 2,5)$ was unstable if it was heated a little above room temperature, but this layer thickness was stable at considerably higher temperature $(T>450 \mathrm{~K})$ if it was formed as a decomposition product of an initially uniform $N+1$ or $N-1$ thick film. This intriguing result suggests that a customary consideration of the energy landscape defined by QW states is incomplete.

In this Letter, we describe low energy electron microscopy (LEEM) investigations of the growth and thermal stability of Ag films on $\mathrm{Fe}(100)$. Measurements of the elastically reflected electron intensity reveal intensity peaks at very low energy $[16,17]$ that are associated with QW resonances above the vacuum level. Highly laterally resolved measurements of $\mathrm{Ag} \mathrm{QW}$ resonances with LEEM are then used to monitor film morphology during annealing and growth. We find that Ag films thermally decompose to thicknesses that are stabilized by QW states at the $\bar{\Gamma}$ point, in agreement with the electronic growth picture. These investigations also reveal the transition of film growth between the kinetically limited and QW electronic growth regimes. Novel kinetically limited electronic growth morphologies are observed that deepen our understanding of the unusual bifurcation mode of thermal decomposition.

The $\mathrm{Fe}(100)$ single crystal substrate was cleaned by extensive Ar ion sputtering and annealing cycles. Ag was deposited from an electron beam heated source at a typical rate of $0.3 \mathrm{ML} / \mathrm{min}$. LEEM imaging was performed using the (00) beam at normal incidence, corresponding to no momentum transfer parallel to the surface. The dependence of the elastically reflected electron intensity upon film thickness and incident electron energy was determined by integrating the LEEM image intensity continuously during deposition at room temperature while repeatedly ramping the incident energy from 0 to $18 \mathrm{eV}$. After normalizing the data by the intensity spec- 
trum of a thick Ag film, intensity peaks are clearly seen that increase in number and disperse towards lower energy with increasing film thickness (Fig. 1). The relationship of these peaks to QW resonances is described by the phase accumulation model, which has been successfully applied in the past to explain QW states below the Fermi level $[18,19]$. According to this model, the quantization condition for the existence of QW states and resonances is $2 k(E) m t+\Phi_{\mathrm{C}}(E)+\Phi_{\mathrm{B}}(E)=2 n \pi$, where $k(E)$ is the perpendicular wave vector of an electron in the film, $m$ is the number of atomic layers, $t$ is the layer spacing, $\Phi_{\mathrm{C}}(E)$ and $\Phi_{\mathrm{B}}(E)$ are the energy dependent phase shifts that occur upon reflection at the interface and surface, respectively, and $n$ is an integer. The quantization condition can be inverted to find an expression for the thickness at which a QW state or resonance exists at a given energy,

$$
m=\frac{\left[\Phi_{\mathrm{C}}(E)+\Phi_{\mathrm{B}}(E)\right] / 2 \pi+\nu}{\kappa(E) / k_{\mathrm{BZ}}},
$$

where the quantum number $\nu=m-n, k_{\mathrm{BZ}}=\pi / t$, and the wave vector $\kappa(E)=k_{\mathrm{BZ}}-k(E)$ is measured from the Brillouin zone boundary in the direction perpendicular to the film plane, i.e., the $X$ point for $\operatorname{Ag}(100)$. For $\operatorname{Ag}(100)$, the $\kappa(E)$ of an $s p$ band with $\Delta_{1}$ symmetry that lies just above the vacuum level can be described well by the two-band, nearly free electron model [19]. We use a formula for the reflection phase that has also been used

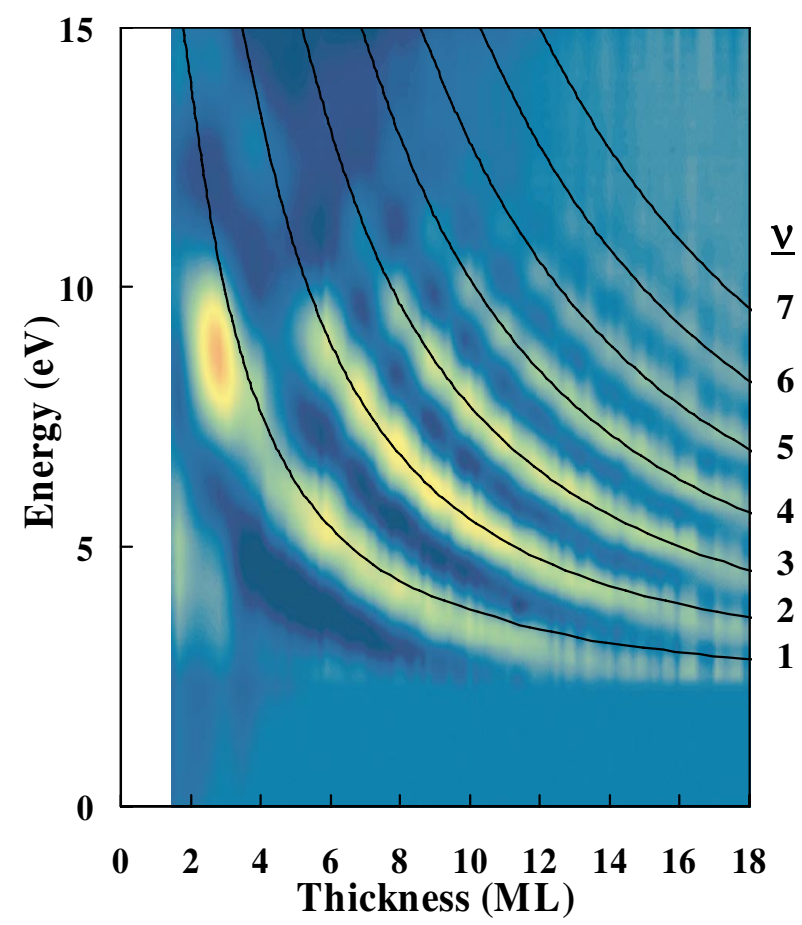

FIG. 1 (color). Integrated LEEM intensity versus incident electron energy and thickness ( $\mathrm{ML}=$ monolayer) for $\mathrm{Ag}$ films grown on $\mathrm{Fe}(100)$ at $300 \mathrm{~K}$. The solid lines through the data are the phase accumulation model predictions for the values of the quantum numbers, $\nu$, that are indicated on the right. previously to understand QW states [18], $\Phi_{\mathrm{C}}(E)=\pi-$ $2 \arcsin \sqrt{\left(E-E_{\mathrm{L}}\right) /\left(E_{\mathrm{U}}-E_{\mathrm{L}}\right)}$, where $E_{\mathrm{L}}=5.0 \mathrm{eV}$ and $E_{\mathrm{U}}=15.5 \mathrm{eV}$ (above the vacuum level) are the lower and upper edge of the Fe band gap, respectively [20]. This band gap is evidenced by experimental observations of a high reflected intensity from the $\mathrm{Fe}(100)$ substrate of the $(00)$ beam at normal incidence in this energy range [21]. We find empirically that the phase shift at the surface is negligible, $\Phi_{\mathrm{B}}(E)=0$.

The resonance conditions calculated using Eq. (1) match the experimental data in Fig. 1 very well using the two-band model parameters that were determined previously from the QW state and bulk photoemission data [19]. These parameters are the pseudopotential form factor, $V=3.033 \mathrm{eV}$, the effective mass, $m^{*}=$ $0.88 m_{e}\left(m_{e}\right.$ is the free electron mass), and the valence band maximum relative to the vacuum level, $E_{\mathrm{vbm}}=$ $-2.679 \mathrm{eV}$, where we have assumed a work function of $\varphi=4.4 \mathrm{eV}$. The excellent agreement validates the use of QW resonances to determine film thickness in subsequent investigations of thermal stability and growth.

The thermal stability of $\mathrm{Ag}$ films that were deposited at $300 \mathrm{~K}$ was studied during stepwise annealing to $500 \mathrm{~K}$. In agreement with previous work [12], 2 and 5 ML films are stable and initially uniform 3 and 4 ML films are unstable during annealing (Fig. 2). However, the 3 and 4 ML films decomposed to stable 2 and 5 ML components, in contrast to the bifurcation mode observed previously. The final decomposition products were determined from laterally

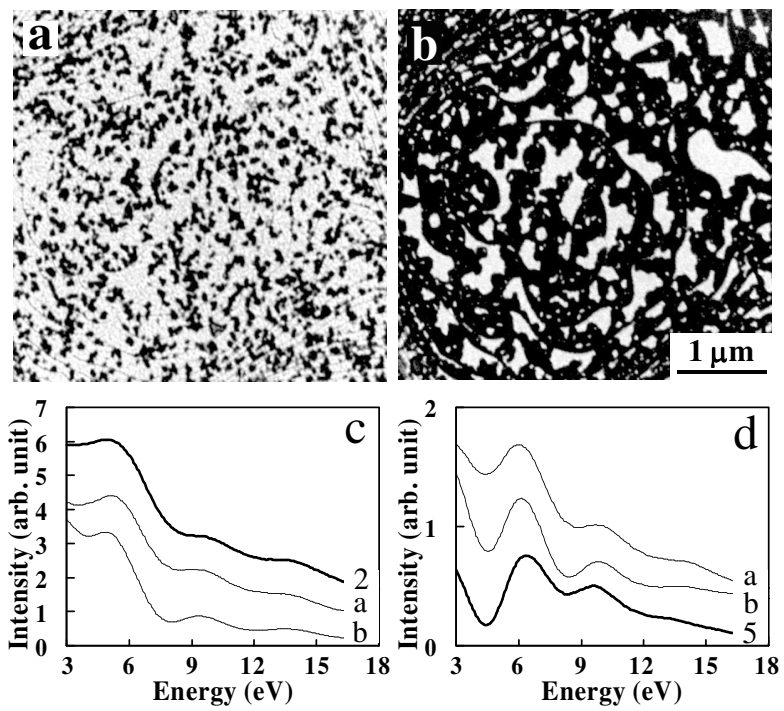

FIG. 2. LEEM images of initially uniform (a) 3 layer and (b) 4 layer Ag films on Fe(100) following thermal decomposition at elevated temperature. The imaging energy is $4.5 \mathrm{eV}$. (c) The local intensity spectra measured in the bright regions in (a) and (b) are compared to the spectrum of the two layer annealed film. (d) The local intensity spectra measured in the dark regions in (a) and (b) are compared to the spectrum of the five layer annealed film. 
resolved intensity spectra measurements of uniform regions in the final morphologies, such as those shown in Fig. 2. The intensity spectra for the bright regions in the images following decomposition of 3 ML [Fig. 2(a)] and 4 ML [Fig. 2(b)] films are shown in comparison to the intensity spectrum for a stable $2 \mathrm{ML}$ annealed film in Fig. 2(c). Similar data for the dark regions in Figs. 2(a) and 2(b) and the intensity spectrum for a stable $5 \mathrm{ML}$ annealed film are shown in Fig. 2(d). These comparisons clearly identify the bright regions in Figs. 2(a) and 2(b) as $2 \mathrm{ML}$ and the dark regions as $5 \mathrm{ML}$. Furthermore, the areas of the 2 and 5 ML regions in Figs. 2(a) and 2(b) are in proportions of $2: 1$ and $1: 2$, respectively. This accounts for all material in the initial $3 \mathrm{ML}[3 \mathrm{ML}=(2 / 3) \times$ $2 \mathrm{ML}+(1 / 3) \times 5 \mathrm{ML}]$ and $4 \mathrm{ML}[4 \mathrm{ML}=(1 / 3) \times$ $2 \mathrm{ML}+(2 / 3) \times 5 \mathrm{ML}]$ films after annealing. Detailed investigations also indicate that the $3 \mathrm{ML}$ film begins to decompose directly to 2 and $5 \mathrm{ML}$ above about $370 \mathrm{~K}$, but the 4 ML film decomposes partially by bifurcation between 320 to $370 \mathrm{~K}$ and then directly to 2 and $5 \mathrm{ML}$ above $370 \mathrm{~K}$ [21]. First principles calculations confirm that morphologies comprising 2 and $5 \mathrm{ML}$ in the observed proportions are energetically favorable compared to the corresponding bifurcation morphologies [22]. These film thicknesses are stabilized by QW states at the $\bar{\Gamma}$ point. Therefore, our experimental results demonstrate that the bifurcation morphologies are metastable configurations. In the previous work [12], where bifurcation was the dominant mode of decomposition, atomically smooth films were prepared initially by deposition at low temperature and annealing to $300 \mathrm{~K}$. Apparently, the small degree of atomic roughness that results from deposition directly at $300 \mathrm{~K}$ in our work is responsible for overcoming the kinetic barriers that stand between the initial or metastable configurations and the observed electronic growth morphologies.

For additional insight into this problem, we have searched for corresponding effects in growth. Figure 1 demonstrates that QW resonances are present for all film thicknesses during growth at $300 \mathrm{~K}$. This is evidence of kinetically limited layer-by-layer growth; i.e., all thicknesses are realized regardless of QW states. On the basis of the thermal decomposition results (Fig. 2), growth of $\mathrm{Ag}$ on $\mathrm{Fe}(100)$ in the absence of kinetic limitations should proceed by the nucleation and lateral growth of triple layer height islands on top of a $2 \mathrm{ML}$ thick wetting layer. This electronic growth morphology is expected to form if the growth temperature is increased above $300 \mathrm{~K}$. In real-time LEEM experiments, we find that a uniform $2 \mathrm{ML} \mathrm{Ag} \mathrm{film} \mathrm{wets} \mathrm{the} \mathrm{surface} \mathrm{and} \mathrm{the} \mathrm{third} \mathrm{layer} \mathrm{deco-}$ rates and grows outward from steps at all temperatures studied between 420 and $475 \mathrm{~K}$ [see Figs. 3(a) and 3(e)] [23]. At $420 \mathrm{~K}$, overgrowth by the fourth layer occurs before completion of the third layer [Fig. 3(b)]. Interestingly, growth of the fourth layer is initiated at

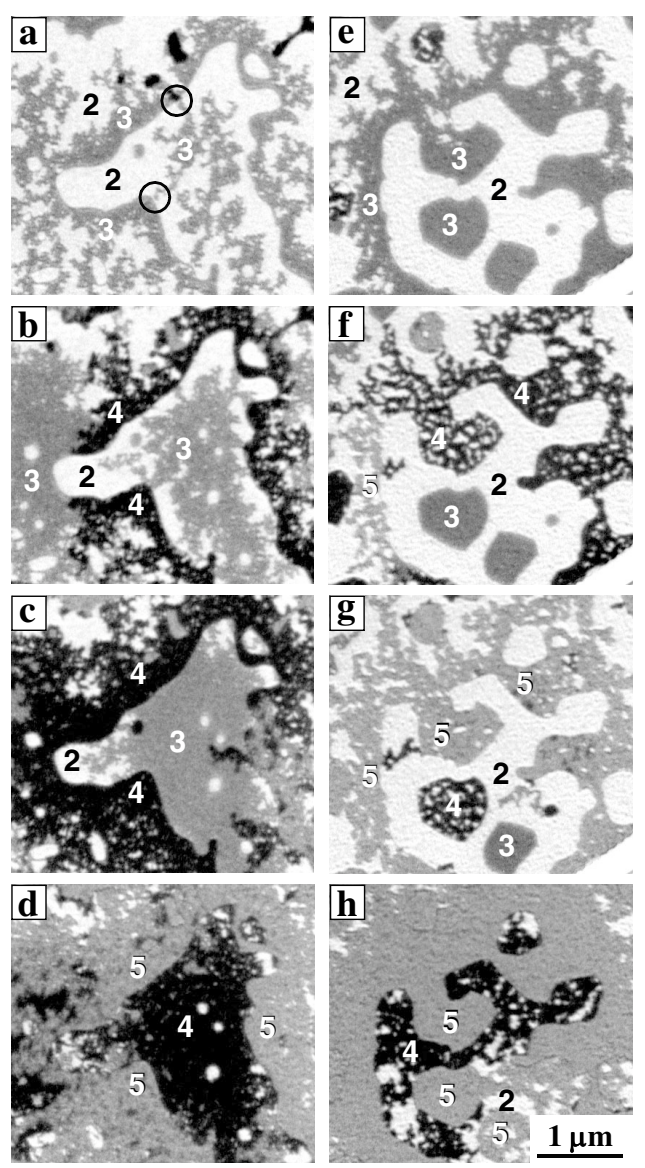

FIG. 3. LEEM images of Ag film growth on Fe(100) at (a)(d) $420 \mathrm{~K}$ and (e) $-(\mathrm{h}) 455 \mathrm{~K}$. The imaging energy is $5.3 \mathrm{eV}$. The film thicknesses of different regions are indicated in monolayer units. See Ref. [23].

positions over the buried interface steps and proceeds along and outward from the buried step position in a step-flow-like manner. This produces an unusual double layer morphological feature that consists of a combination of a single interface step and an inverted surface step, shown schematically in Fig. 4(a). We also find that a novel double layer growth occurs when the fourth layer reaches the more slowly advancing third layer growth front [Figs. 3(b) and 3(c)]. Unlike double layer electronic growth in other systems $[3,6-8,10]$, double layer growth ensues here for a total $\mathrm{Ag}$ thickness [4 ML in this case, shown schematically in Fig. 4(a)] that is not associated with a QW-induced minimum of the energy. This double layer growth and novel inverted step [Fig. 4(a)] are therefore analogous to the (kinetically limited) bifurcation mode of decomposition of $3 \mathrm{ML}$ into 2 and 4 ML. With further growth at $420 \mathrm{~K}$, the inverted step may be annihilated by the advance of surviving three layer film regions. This leads to the coverage of the surface with a combination of 3 and 4 ML regions separated by a buried interface step [Fig. 3(c)]. A single layer then grows uniformly until the surface is covered by a combination of 4 


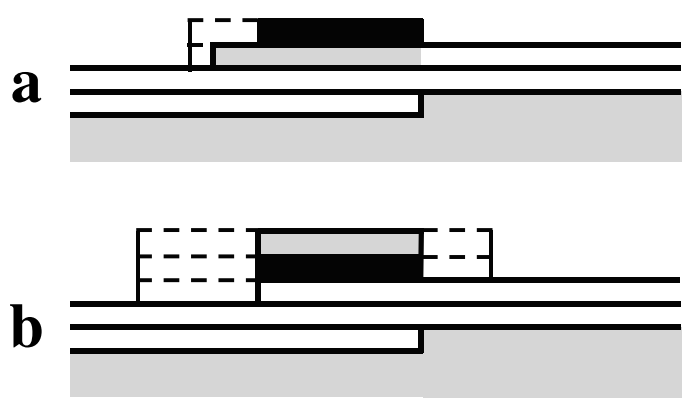

FIG. 4. Schematic drawings of morphologies observed during growth of $\mathrm{Ag}$ on $\mathrm{Fe}(100)$. The shading in this figure corresponds to the intensity of corresponding features in Fig. 3. The dashed sections indicate double and triple layer growth.

and 5 ML regions [Fig. 3(d)]. Subsequent growth occurs only on the energetically unfavorable $4 \mathrm{ML}$ regions until a uniform $5 \mathrm{ML}$ film covers the surface.

There are several important differences when growth is carried out at higher temperature [23]. At $455 \mathrm{~K}$, lateral growth of the fourth layer over the third layer is completely overwhelmed by a rapid decomposition of the 3 ML regions [Fig. 3(e)] into a combination of 2 and $4 \mathrm{ML}$ (bifurcation) or even directly into 2 and $5 \mathrm{ML}$ [Fig. 3(f)] regions. Decomposition is obvious because of intensity changes and the formation of two or three layer deep holes in these regions that conserve material locally. This generates the single inverted step [Fig. 4(a)] or a new inverted double surface step [Fig. 4(b)] at the buried interface step positions. The 4 and 5 ML thick regions then grow outward from steps and the holes begin to fill in by the advancement of double [Fig. 4(a)] and triple layer high steps [Fig. 4(b)], respectively. Meanwhile, the $4 \mathrm{ML}$ regions also decompose rapidly into a combination of 2 and $5 \mathrm{ML}$ regions [Fig. 3(g)] and then outward growth from steps occurs exclusively by advancement of triple layer high steps [Fig. 3(h)]. Double layer growth is also initiated at the inverted double step [Figs. 3(h) and 4(b)], but the $4 \mathrm{ML}$ regions that this produces soon decompose to 2 and $5 \mathrm{ML}$. Then, triple layer height growth proceeds over the entire surface. The phenomena observed at $455 \mathrm{~K}$ are reproduced at $475 \mathrm{~K}$, although the electronic triple layer growth on top of the $2 \mathrm{ML}$ wetting film becomes even more dominant [23].

The thermal decomposition results presented here suggest that adatom-vacancy formation kinetics may limit electronic growth and promote bifurcation. Real-time LEEM measurements also reveal that analogous bifurcation and electronic growth morphologies develop rapidly when $\mathrm{Ag}$ adatoms are introduced during deposition. However, the crucial observation is that double and triple layer morphological features seldom nucleate spontaneously during growth, but are formed most often when an advancing single or double layer passes over a buried interface step. This is demonstrated, for example, by the circled features in Fig. 3(a). Therefore, nucleation of multiple layer high islands is identified as the process that limits electronic growth.

In summary, we have presented compelling evidence that $\mathrm{Ag}$ films on $\mathrm{Fe}(100)$ decompose to thicknesses stabilized by QW states at the $\bar{\Gamma}$ point, and therefore that consideration of kinetic limitations and QW states is essential for understanding the unusual bifurcation mode of thermal decomposition that was reported for this system previously. Novel kinetically limited growth morphologies were also observed that have not yet been reported for systems subject to the influence of QW states. Nevertheless, related phenomena should occur in an appropriate temperature range for all systems that exhibit electronic growth. These results indicate that kinetic limitations enrich electronic growth phenomena and therefore may offer an additional means to manipulate film morphology.

This work was supported by the Hong Kong Research Grants Council under Grant No. HKUST600103.

[1] T. C. Chiang, Surf. Sci. Rep. 39, 181 (2000).

[2] S. S. Parkin et al., Phys. Rev. Lett. 64, 2304 (1990).

[3] B. J. Hinch et al., Europhys. Lett. 10, 341 (1989).

[4] A. R. Smith et al., Science 273, 226 (1996).

[5] Z. Zhang et al., Phys. Rev. Lett. 80, 5381 (1998).

[6] L. Gavioli et al., Phys. Rev. Lett. 82, 129 (1999).

[7] V. Yeh et al., Phys. Rev. Lett. 85, 5158 (2000).

[8] W. B. Su et al., Phys. Rev. Lett. 86, 5116 (2001).

[9] H. Yu et al., Phys. Rev. Lett. 88, 016102 (2002).

[10] A. Menzel et al., Phys. Rev. B 67, 165314 (2003).

[11] H. Hong et al., Phys. Rev. Lett. 90, 076104 (2003).

[12] D. A. Luh et al., Science 292, 1131 (2001).

[13] C. M. Wei and M.Y. Chou, Phys. Rev. B 68, 125406 (2003).

[14] J. J. Paggel et al., Phys. Rev. B 66, 233403 (2002).

[15] A. G. Danese et al., Phys. Rev. B 70, 165420 (2004).

[16] W. F. Chung et al., Phys. Rev. Lett. 90, 216105 (2003).

[17] R. Zbyd and E. Bauer, Phys. Rev. Lett. 88, 166403 (2002).

[18] N.V. Smith et al., Phys. Rev. B 49, 332 (1994).

[19] J. J. Paggel et al., Phys. Rev. B 61, 1804 (2000).

[20] R. Bertacco and F. Ciccacci, Phys. Rev. B 59, 4207 (1999).

[21] K. L. Man, M. S. Altman, and Z. Q. Qiu (to be published).

[22] M. Y. Chou and C. M. Wei (private communication).

[23] See EPAPS Document No. E-PRLTAO-93-051446 for LEEM videos of Ag growth on $\mathrm{Fe}(100)$ corresponding to Fig. 3. A direct link to this document may be found in the online article's HTML reference section. The document may also be reached via the EPAPS homepage (http://www.aip.org/pubservs/epaps.html) or from ftp.aip.org in the directory /epaps/. See the EPAPS homepage for more information. 